\title{
Analytical Solutions of Vortex Rossby Waves in a Discrete Barotropic Model
}

\author{
Takahiro ITO \\ Numerical Prediction Division, Japan Meteorological Agency, Tokyo, Japan \\ and \\ Hirotada KANEHISA \\ Meteorological College, Kashiwa, Japan
}

(Manuscript received 1 February 2013, in final form 11 August 2013)

\begin{abstract}
The initial value problem of vortex Rossby waves (VRWs) is analytically solved in a linearized barotropic system on an $f$ plane. The basic axisymmetric vorticity $\bar{q}$ is assumed to be piecewise uniform in the radial direction so that the radial gradient $\frac{d \bar{q}}{d r}$ and the disturbance vorticity $q$ are expressed in terms of Dirac delta functions. After Fourier transformation in the azimuthal direction with the wavenumber $m$, the linearized vorticity equation becomes a system of ordinary differential equations with respect to time; these can be analytically solved to give a closed-form solution with a prescribed initial value.

For a monopolar $\bar{q}$, the solution of $q$ starting from the innermost radius exhibits the outward propagation of VRWs. As the outer disturbances are generated, the inner disturbance is diminished. On the other hand, in the case of a solution forced at the innermost radius, the inner disturbance is not diminished, and the outward propagation of VRWs forms a distribution of spiral-shaped disturbance vorticity.

For a basic vorticity $\bar{q}$ with a moat, and if the radial distribution of $\bar{q}$ satisfies a certain additional condition, the solution of $q$ with $|m| \neq 1$ grows exponentially or linearly in time as a result of the interaction of counterpropagating VRWs near the moat. Although the solution of $q$ with $|m|=1$ cannot grow exponentially for any $\bar{q}$, it can grow as a linear function of time. This linear growth may be regarded as a result of resonance between two internal modes of the system.
\end{abstract}

Keywords vortex Rossby wave; spiral band; linearly growing disturbance

\section{Introduction}

For cases in which the radial gradient $\frac{d \bar{q}}{d r}$ of a basic axisymmetric vorticity $\bar{q}$ is present, vortex Rossby waves (VRWs) are present. The vorticity disturbance $q$ added to $\bar{q}$ induces a circulation around it, which is cyclonic [anticyclonic] if $q>0[q<0]$. The cyclonic [anticyclonic] circulation advects $\bar{q}$ to generate a new positive [negative] $q$ to the left of the original $q$

Corresponding author: Takahiro Ito, Numerical Prediction Division, Japan Meteorological Agency, 1-3-4 Otemachi, Chiyodaku, Tokyo 100-8122, Japan

E-mail: t ito@met.kishou.go.jp

C2013, Meteorological Society of Japan pointing in the direction of $\frac{d \bar{q}}{d r}$. As a result, $q$ azimuthally propagates to the left of $\frac{d \bar{q}}{d r}$. This is the VRW. Because the circulation induced by $q$ advects $\bar{q}$ also at different radii, the VRW propagates radially and azimuthally, resulting in diagonal (i.e., spiral) propagation. Here and hereafter, "propagation" means the propagation relative to the fluid. If $\frac{d \bar{q}}{d r}$ is not signdefinite, e.g., positive at $r^{\prime}$ and negative at $r^{\prime \prime}$, then the VRWs at $r^{\prime}$ and $r^{\prime \prime}$ propagate azimuthally counter to each other. Further, if a certain additional condition is satisfied, the VRWs can interact so that the circulation induced by $q$ at $r^{\prime}$ reinforces $q$ at $r^{\prime \prime}$ and vice versa. As a result, the VRWs grow exponentially in time (e.g., Hoskins et al. 1985). 
The abovementioned propagation and interaction of VRWs are now well known, and many authors studied several aspects of tropical cyclones in terms of VRWs. Montgomery and Kallenbach (1997) presented a theory for the propagation and interaction of VRWs with a basic vortex. They analyzed the effects of radially propagating VRWs on the basic vortex. Schubert et al. (1999) examined the polygonal eyewalls of tropical cyclones. They analytically and numerically showed that the annular vorticity ring at the eyewall supports counterpropagating VRWs; depending on the vorticity structure of the ring, they showed that the VRWs grow exponentially to generate the polygonal eyewall. Chen and Yau (2001) analyzed the potential vorticity spiral bands of a numerically simulated tropical cyclone. They showed that the propagation properties are consistent with the VRW theory. Wang (2002) analyzed the asymmetric inner core structure of a numerically simulated tropical cyclone. He found that the asymmetry is dominated by the VRWs of the azimuthal wavenumbers $m=1$ and 2 .

In addition to the problem of spiral bands and polygonal eyewalls, the resiliency of a tropical cyclone to environmental vertical shear was also considered in terms of VRWs (e.g., Schecter et al. 2002; Mallen and Montgomery 2005). A tropical cyclone in an environmental flow with vertical shear resists the shear and maintains its upright vertical structure if the shear is not so strong. The VRWs with $m=1$ of the tropical cyclone, which are generated by vertical shear, are supposed to participate in the resiliency.

Reznik and Dewar (1994) showed that with $m=1$, disturbances cannot grow exponentially in a linearized barotropic model. However, as the exact solution derived by Smith and Rosenbluth (1990) shows, disturbances with $m=1$ grow asymptotically as a function of $t^{1 / 2}$ as $t \rightarrow \infty$ if the basic angular velocity has a maximum radius other than the center. Nolan and Montgomery (2000) further investigated the algebraic growth of $m=1$ disturbances for hurricane-like vortices.

In spite of many studies on VRWs, their analytical solutions have not yet been obtained, except for the solution of $m=1$ in Smith and Rosenbluth (1990), at least to our knowledge. In this paper, we analytically solve the initial value problem of VRWs in a linearized discrete barotropic model on an $f$ plane.

The discrete model has $N+1(\geq 2)$ regions in the radial direction, and in each of which, the vorticity is uniform. As is already known, disturbances with $m=$ 1,2 cannot grow in the usual three-region model, which has an inner region with low positive vorticity, an annulus with high positive vorticity, and an environment with zero vorticity (e.g., Flierl 1988; Schubert et al. 1999; Terwey and Montgomery 2002). However, it has been shown that they can grow in an $[N+1(\geq 4)]$ region model.

Further, the obtained solutions exhibit some new aspects. For $m \neq 1$ (and if the radial distribution of $\bar{q}$ satisfies a certain condition), the VRW grows as a linear function of time, which occurs as a marginal solution between stable and exponentially unstable solutions. Also, for $m=1$, the linear marginal growth of VRWs can occur, although exponential growth is impossible for any $\bar{q}$.

This paper is organized as follows. In Section 2, the governing equation of disturbance vorticity in a discrete barotropic system is derived. In Section 3, the analytical solution of the governing equation with a prescribed initial value is presented. In Section 4, stable solutions are considered. In Sections 5 and 6, exponentially and linearly growing solutions are considered, respectively. In Section 7, concluding remarks are given.

\section{Governing equation}

We begin with the following barotropic vorticity equation on an $f$ plane linearized about an axisymmetric basic vorticity $\bar{q}=\bar{q}(r)$ with azimuthal velocity $\bar{v}=$ $\bar{v}(r)$ :

$$
\frac{\partial q}{\partial t}+\frac{\bar{v}}{r} \frac{\partial q}{\partial \theta}-\frac{1}{r} \frac{d \bar{q}}{d r} \frac{\partial \psi}{\partial \theta}=\dot{q}
$$

where $t, r$, and $\theta$ are the temporal, radial, and azimuthal coordinates, respectively, $q=q(t, r, \theta)=\frac{\partial^{2} \psi}{\partial r^{2}}+\frac{1}{r} \frac{\partial \psi}{\partial r}$ $+\frac{1}{r^{2}} \frac{\partial^{2} \psi}{\partial \theta^{2}}$ and $\psi=\psi(t, r, \theta)$ are the disturbance vorticity and stream function, respectively, and $\dot{q}=\dot{q}(t, r, \theta)$ represents forcing. Fourier transformation (2.1) with respect to $\theta$ with the wavenumber $m$ and expression of the Fourier transform $\hat{\psi}$ of $\psi$ in terms of the Green's function $G\left(r, r^{\prime}\right)$ as $\hat{\psi}(t, r)=\int_{0}^{\infty} d r^{\prime} \mathrm{r}^{\prime} \mathrm{G}\left(r, r^{\prime}\right) \hat{q}\left(t, r^{\prime}\right)$ give

$$
\begin{aligned}
& \frac{\partial \hat{q}(t, r)}{\partial t}+i m \frac{\bar{v}}{r} \hat{q}(t, r) \\
& -i m \frac{1}{r} \frac{d \bar{q}}{d r} \int_{0}^{\infty} d r^{\prime} r^{\prime} G\left(r, r^{\prime}\right) \hat{q}\left(t, r^{\prime}\right)=\hat{\dot{q}}(t, r) .
\end{aligned}
$$

Here $\hat{q}(t, r)=\hat{q}(t, r, m)$ is the Fourier transform of $q(t, r$, $\theta)$. Since $\left(\frac{1}{r} \frac{\partial}{\partial r} r \frac{\partial}{\partial r}-\frac{m^{2}}{r^{2}}\right) \hat{\psi}=\hat{q}$ with $\lim _{r \rightarrow 0, \infty} \hat{\psi}=0$, the Green's function in (2.2) is given by

$$
\begin{aligned}
& G\left(r, r^{\prime}\right)=-\frac{1}{2|m|} g\left(r, r^{\prime}\right), \\
& \text { where } g\left(r, r^{\prime}\right)=\left(\frac{r}{r^{\prime}}\right)^{|m|} \quad \text { if } \quad r<r^{\prime},
\end{aligned}
$$




$$
g\left(r, r^{\prime}\right)=\left(\frac{r^{\prime}}{r}\right)^{|m|} \quad \text { if } \quad r^{\prime}<r .
$$

The basic vorticity $\bar{q}(r)$ is assumed to be piecewise uniform in the radial direction (see Fig. 1),

$$
\begin{aligned}
& \bar{q}(r)=\sum_{k=j}^{N+1} \bar{q}_{k} \\
& \text { for } \quad r_{j-1}<r<r_{j}, \quad j=1,2, \ldots, N, N+1,
\end{aligned}
$$

where $0=r_{0}<r_{1}<r_{2}<\cdots<r_{N}<r_{N+1}=\infty$ and $\bar{q}_{N+1}=0$, so that $\frac{d \bar{q}(r)}{d r}$ is expressed in terms of Dirac delta functions. The forcing term is also assumed to be of the same form:

$$
\begin{gathered}
\frac{d \bar{q}(r)}{d r}=-\sum_{j=1}^{N} \bar{q}_{j} \delta\left(r-r_{j}\right), \\
\hat{\dot{q}}(t, r)=\sum_{j=1}^{N} \hat{\dot{q}}_{j}(t) \delta\left(r-r_{j}\right) .
\end{gathered}
$$

From (2.5) and (2.6), and under the assumption of the absence of the initial disturbance vorticity except at $r=$ $r_{j}, j=1,2, \ldots, N$, the disturbance vorticity can be written as

$$
\hat{q}(t, r)=\sum_{j=1}^{N} \hat{q}_{j}(t) \delta\left(r-r_{j}\right) .
$$

With the redefinition of $\tilde{q}_{j}(t)=r \hat{q}_{j}(t)$, and by substitution of (2.3), (2.5), (2.6), and (2.7) into (2.2), we obtain the following governing equation:

$$
\begin{aligned}
& \frac{\partial \tilde{q}_{j}(t)}{\partial t}+i m \bar{\omega}_{j} \tilde{q}_{j}(t)-i \frac{m}{2|m|} \bar{q}_{j} \sum_{k=1}^{N} g_{j k} \tilde{q}_{k}(t)=\tilde{\dot{q}}_{j}(t), \\
& j=1,2, \ldots, N .
\end{aligned}
$$

Here $g_{j k}=g\left(r_{j}, r_{k}\right)$ and $\bar{\omega}_{j}=\bar{\omega}\left(r_{j}\right)=\frac{\bar{v}\left(r_{j}\right)}{r_{j}}$. From $\bar{q}=\frac{1}{r} \frac{d(r \bar{v})}{d r}$ and the piecewise-uniform assumption of $\bar{q}$ in (2.4), the basic angular velocity $\bar{\omega}(r)=\frac{\bar{v}(r)}{r}$ is given by

$$
\begin{aligned}
& \bar{\omega}(r)=\frac{1}{2} \sum_{k=j}^{N} \bar{q}_{k}+\frac{1}{2} \sum_{k=1}^{j-1}\left(\frac{r_{k}}{r}\right)^{2} \bar{q}_{k} \\
& \text { for } \quad r_{j-1} \leq r \leq r_{j}, \quad j=1,2, \ldots, N+1 .
\end{aligned}
$$

The governing Eq. (2.8) can be rewritten as the following vector form:

$$
\frac{\partial|\tilde{q}(t)\rangle}{\partial t}+i m \Lambda|\tilde{q}(t)\rangle=|\tilde{\dot{q}}(t)\rangle,
$$

where $|\tilde{q}(t)\rangle$ denotes the $N$-component column vector whose $j$ th component is $\tilde{q}_{j}(t)$, and $\Lambda$ denotes the $N \times N$ matrix whose $(j, k)$ th component is

$$
\Lambda_{j k}=\bar{\omega}_{j} \delta_{j k}-\frac{1}{2|m|} \bar{q}_{j} g_{j k} .
$$

The diagonal component of (2.11) $\Lambda_{j j}=\bar{\omega}_{j}-\frac{1}{2|m|} \bar{q}_{j}$

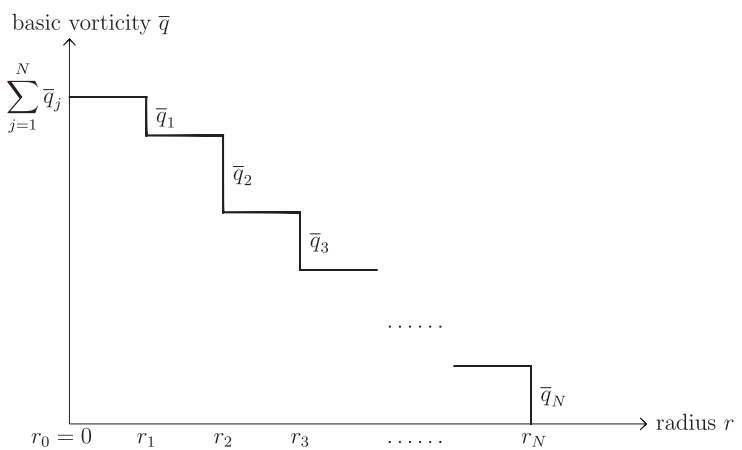

Fig. 1. Radial distribution of basic vorticity in the $N+1$ region model.

represents the free angular phase velocity of the disturbance at $r_{j}$. Here the free angular phase velocity means the angular phase velocity without the effects from the other disturbances at $r_{k} \neq r_{j}$. The first term $\bar{\omega}_{j}$ of $\Lambda_{j j}$ represents the advection by the basic flow $\bar{v}$; the second term $-\frac{1}{2|m|} \bar{q}_{j}$ of $\Lambda_{j j}$ represents the propagation owing to the radial gradient of the basic vorticity $\bar{q}$.

\section{Analytical solution}

The solution to (2.10) with a prescribed initial value $|\tilde{q}(0)\rangle$ is given by the following formula:

$$
\begin{aligned}
|\tilde{q}(t)\rangle= & \sum_{n=1}^{N} e^{-i m \lambda_{n} t} \frac{\left|R_{n}\right\rangle\left\langle L_{n}\right|}{\left\langle L_{n} \mid R_{n}\right\rangle}|\tilde{q}(0)\rangle \\
& +\sum_{n=1}^{N} e^{-i m \lambda_{n} t} \int_{0}^{t} d t^{\prime} e^{i m \lambda_{n} t^{\prime}} \frac{\left|R_{n}\right\rangle\left\langle L_{n}\right|}{\left\langle L_{n} \mid R_{n}\right\rangle}\left|\tilde{\dot{q}}\left(t^{\prime}\right)\right\rangle
\end{aligned}
$$

where $\lambda_{n}$ is the $n$th eigenvalue of the matrix $\Lambda$, the column vector $\left|R_{n}\right\rangle$ is the right eigenvector for the eigenvalue $\lambda_{n}$, i.e., $\Lambda\left|R_{n}\right\rangle=\lambda_{n}\left|R_{n}\right\rangle$, and $\left\langle L_{n}\right|$, which denotes an $N$-component row vector, is the left eigenvector for $\lambda_{n}$, i.e., $\left\langle L_{n}\right| \Lambda=\lambda_{n}\left\langle L_{n}\right|$. In (3.1), $\left\langle L_{n} \mid R_{n}\right\rangle=\sum_{j=1}^{N} L_{n j} R_{n j}$ is the scalar product of $\left\langle L_{n}\right|$ and $\left|R_{n}\right\rangle .\left|R_{n}\right\rangle\left\langle L_{n}\right|$ is the diad product of $\left|R_{n}\right\rangle$ and $\left\langle L_{n}\right|$ whose $(j, k)$ th component is $R_{n j} L_{n k}$. From the definition of eigenvectors and the completeness relation $\sum_{n=1}^{N} \frac{\left|R_{n}\right\rangle\left\langle L_{n}\right|}{\left\langle L_{n} \mid R_{n}\right\rangle}$ $=1$, it is easily seen that $(3.1)$ is indeed the solution to (2.10) with the prescribed $|\tilde{q}(0)\rangle$. The first term on the right-hand side (RHS) of (3.1) is the free solution starting from $|\tilde{q}(0)\rangle \neq 0$ with $|\tilde{\dot{q}}(\mathrm{t})\rangle=0$. The second term is the forced solution starting from a null initial value $|\tilde{q}(0)\rangle=0$ with $|\tilde{\dot{q}}(\mathrm{t})\rangle \neq 0$. For mathematical simplicity, we assume that the initial value $|\tilde{q}(0)\rangle$ is real and that the forcing term has the form $|\tilde{\dot{q}}(\mathrm{t})\rangle=$ $e^{-i \Omega t}|\tilde{\dot{q}}(0)\rangle$ with $|\tilde{\dot{q}}(0)\rangle$ being real.

If the basic vortex is a simple monopole, i.e., $\bar{q}_{j}$ in (2.5) is positive for any $j=1,2, \ldots, N$, the Rayleigh 
condition is not satisfied, and then the solution (3.1) cannot grow exponentially. This implies that $\lambda_{n},\left|R_{n}\right\rangle$, and $\left\langle L_{n}\right|$ are real for any $n=1,2, \ldots, N$. In this case, inversely Fourier transforming (3.1) [i.e., multiplying by $e^{i m \theta}$ and taking the real part] gives the following monochromatic solution with wavenumber $m$ in the physical space $(t, \theta)$ :

$$
\begin{aligned}
& |r q(t, \theta)\rangle=\sum_{n=1}^{N} \cos m\left(\theta-\lambda_{n} t\right) \cdot \frac{\left|R_{n}\right\rangle\left\langle L_{n}\right|}{\left\langle L_{n} \mid R_{n}\right\rangle}|\tilde{q}(0)\rangle \\
& +\sum_{n=1}^{N} \frac{\sin (m \theta-\Omega t)-\sin m\left(\theta-\lambda_{n} t\right)}{m \lambda_{n}-\Omega} \cdot \frac{\left|R_{n}\right\rangle\left\langle L_{n}\right|}{\left\langle L_{n} \mid R_{n}\right\rangle}|\tilde{q}(0)\rangle,
\end{aligned}
$$

where $|r q(t, \theta)\rangle$ is the column vector whose $j$ th component is $r_{j} q_{j}(t, \theta)$.

However, if the basic vortex has at least one moat, i.e., $\bar{q}_{j}$ in (2.5) is negative for some $j=1,2, \ldots, N$, the Rayleigh condition is satisfied. Then, the solution (3.1) may grow exponentially if a certain additional criterion is satisfied. The additional criterion is a condition on the radial distribution of $\bar{q}$ since the matrix $\Lambda$ in (2.10) depends on $|m|$ and $\left\{\bar{q}_{j}\right.$ and $r_{j}, j=1$, $2, \ldots, N\}$ from (2.3), (2.9), and (2.11). In this case, $\left\{\lambda_{n^{\prime}}\right.$, $\left.\left|R_{n^{\prime}}\right\rangle,\left\langle L_{n^{\prime}}\right|\right\}$ and $\left\{\lambda_{n^{\prime \prime}},\left|R_{n^{\prime \prime}}\right\rangle,\left\langle L_{n^{\prime \prime}}\right|\right\}$ (or more pairs) are complex conjugate to each other for some $n^{\prime}, n^{\prime \prime}=1$, $2, \ldots, N$. In the case of one complex-conjugate pair $\left(n^{\prime}, n^{\prime \prime}\right)$, inversely Fourier transforming (3.1) with $|\tilde{\dot{q}}(t)\rangle=0$ gives the following exponentially growing free monochromatic solution with wavenumber $m$ in the physical space $(t, \theta)$ :

$$
\begin{aligned}
& |r q(t, \theta)\rangle= \\
& 2 \cosh m \lambda_{I} t \cdot \cos m\left(\theta-\lambda_{R} t\right) \cdot\left[\frac{|R\rangle\langle L|}{\langle L \mid R\rangle}\right]_{R}|\tilde{q}(0)\rangle \\
& -2 \sinh m \lambda_{l} t \cdot \sin m\left(\theta-\lambda_{R} t\right) \cdot\left[\frac{|R\rangle\langle L|]}{\langle L \mid R\rangle}\right]_{I}|\tilde{q}(0)\rangle \\
& +\sum_{n \neq n^{\prime}, n^{\prime \prime}} \cos m\left(\theta-\lambda_{n} t\right) \cdot \frac{\left|R_{n}\right\rangle\left\langle L_{n}\right|}{\left\langle L_{n} \mid R_{n}\right\rangle}|\tilde{q}(0)\rangle,
\end{aligned}
$$

where $\lambda_{R}$ and $\lambda_{I}$ are the real and imaginary parts of $\lambda_{n^{\prime}}$, respectively, and $\left[\frac{[R\rangle\langle L|}{\langle L \mid R\rangle}\right]_{R}$ and $\left[\frac{[R\rangle\langle L|}{\langle L \mid R\rangle}\right]$, are the real and imaginary parts of $\frac{\left|R_{n}\right\rangle}{\left\langle n_{n} \mid L_{n}\right\rangle}$, respectively. [If the forcing term $|\tilde{q}(t)\rangle \neq 0$ is included, the forced part is of course added to (3.3).]

In addition to the exponential growth, the solution (3.1) may grow as a linear function of time. This occurs in two cases. The first case is if the angular frequency $\Omega$ of the forcing term $|\tilde{\dot{q}}(t)\rangle=e^{-i \Omega t}|\tilde{\dot{q}}(0)\rangle$ is equal to $m \lambda_{n}$ for some $n$. In this case, as is easily seen, the second term on the RHS of (3.1) grows as a linear function of time. In other words, the internal mode $e^{-i m \lambda_{n} t}$ resonates with a forcing that has the same angular frequency.

The second case is if two eigenvalues $\lambda_{n^{\prime}}$ and $\lambda_{n^{\prime \prime}}$ degenerate into one. In this case, the first term on the RHS of (3.1) grows as a linear function of time (see Section 6).

\section{Stable solution}

In this section, we consider the case of a monopolar basic vortex (see Fig. 1). For $N=1$ (the Rankine vortex), the vectors are reduced to corresponding scalars, i.e., $\left|R_{1}\right\rangle=1,\left\langle L_{1}\right|=1$, and $|r q(t, \theta)\rangle=r_{1} q_{1}(t$, $\theta$ ), and the stable solution (3.2) becomes the following single equation with $\lambda_{1}=\frac{\bar{q}_{1}}{2}-\frac{\bar{q}_{1}}{2|m|}$ :

$$
\begin{aligned}
& r_{1} q_{1}(t, \theta)=\cos m\left(\theta-\lambda_{1} t\right) \cdot \tilde{q}_{1}(0) \\
& +\frac{\sin (m \theta-\Omega t)-\sin m\left(\theta-\lambda_{1} t\right)}{m \lambda_{1}-\Omega} \cdot \tilde{\dot{q}}_{1}(0) .
\end{aligned}
$$

The first term on the RHS of (4.1) is the free solution with $\tilde{q}_{1}(0)=0$ starting from the prescribed initial value $\tilde{q}_{1}(0) \neq 0$. The first term $\frac{\bar{q}_{1}}{2}$ in $\lambda_{1}$ represents counterclockwise advection by the basic flow, and the second term $-\frac{\bar{q}_{1}}{2|m|}$ in $\lambda_{1}$ represents clockwise propagation as the VRW. Except for $|m|=1$, in which case the free solution is stationary, advection dominates the propagation, and the free solution rotates counterclockwise.

The second term on the RHS of (4.1) is the forced solution with $\tilde{\dot{q}}_{1}(0) \neq 0$ starting from a null initial value $\tilde{q}_{1}(0)=0$. In particular, if the angular frequency $\Omega$ of the forcing term is equal to $m \lambda_{1}$, the forced solution grows as a linear function of time:

$$
\text { forced part of } r_{1} q_{1}(\theta, t)=t \cdot \cos m\left(\theta-\lambda_{1} t\right) \cdot \tilde{\dot{q}}_{1}(0) \text {. }
$$

For $N=2$, the eigenvalues of the matrix $\Lambda$ in (2.10) become

$$
\begin{aligned}
& \lambda_{1,2}=\frac{\bar{q}_{1}}{4}\left\{1+2 \eta+\varepsilon^{2}-\frac{1+\eta}{|m|} \pm \sqrt{D}\right\} \text { with } \\
& D=\left(1-\varepsilon^{2}-\frac{1-\eta}{|m|}\right)^{2}+\frac{4 \varepsilon^{2|m|}}{|m|^{2}} \eta,
\end{aligned}
$$

where $\eta=\frac{\bar{q}_{2}}{\bar{q}_{1}}$ and $\varepsilon=\frac{r_{1}}{r_{2}}$. The right and left eigenvectors are, respectively, given by

$$
\begin{aligned}
\left|R_{1,2}\right\rangle & =\left[\begin{array}{c}
\frac{\varepsilon^{|m|}}{|m|} \\
1+\eta-\frac{1}{|m|}-\lambda_{1,2}
\end{array}\right] \text { and } \\
\left\langle L_{1,2}\right| & =\left[\begin{array}{ll}
\frac{\varepsilon^{|m|}}{|m|} \eta & 1+\eta-\frac{1}{|m|}-\lambda_{1,2}
\end{array}\right] .
\end{aligned}
$$

As is expected, the discriminant $D$ in (4.3) is positive 
definite for the monopolar vortex $\eta=\frac{\bar{q}_{2}}{\bar{q}_{1}}>0$ so that the eigenvalues $\lambda_{1}$ and $\lambda_{2}$ are real. In this case, the stable solution (3.2) is reduced to the following twocomponent equation:

$$
\begin{aligned}
& {\left[\begin{array}{l}
r_{1} q_{1}(t, \theta) \\
r_{2} q_{2}(t, \theta)
\end{array}\right]=\sum_{n=1}^{2} \cos m\left(\theta-\lambda_{n} t\right) \cdot \frac{\left|R_{n}\right\rangle\left\langle L_{n}\right|}{\left\langle L_{n} \mid R_{n}\right\rangle}|\tilde{q}(0)\rangle} \\
& +\sum_{n=1}^{2} \frac{\sin (m \theta-\Omega t)-\sin m\left(\theta-\lambda_{n} t\right)}{m \lambda_{n}-\Omega} \cdot \frac{\left|R_{n}\right\rangle\left\langle L_{n}\right|}{\left\langle L_{n} \mid R_{n}\right\rangle}|\tilde{q}(0)\rangle .
\end{aligned}
$$

The solution for $N=2$ in (4.5) exhibits radial propagation and azimuthal propagation.

In the discrete model, VRWs exist only on the edges of uniform vorticity regions. Thus, the VRWs must remain on the edges, and they cannot actually propagate in the radial direction. Rather, the VRWs are successively generated on the edges, from one edge to the next. However, we believe that the successive generation in the discrete model reflects at least some aspect of the radial propagation in the continuous model. Hence, we call the successive generation radial propagation.

For $N=2$, radial propagation is not easily seen because of the reflection between $r_{1}$ and $r_{2}$. Hence, for a clear representation of radial propagation, the free solution [i.e., the first term on the RHS of (3.2)] and the forced solution (i.e., the second term) for $m=2$, $N=5$ are shown in Figs. 2 and 3, respectively. The free solution in Fig. 2 starts from a nonzero initial value at the innermost radius $r_{1}$. The forced solution in Fig. 3 starts from a null initial value, and the forcing term $|\tilde{\dot{q}}(t)\rangle$ is assumed to have an amplitude only at the innermost radius $r_{1}$. The innermost forcing is assumed to have the innermost free angular phase velocity $\Lambda_{11}$, i.e., $\tilde{\dot{q}}_{1}(t)=\mathrm{e}^{-i m \Lambda_{11} t} \tilde{\dot{q}}_{1}(0)$, to mimic the forcing of eyewall convection. In Fig. 2, the innermost disturbance decreases as the VRW diagonally propagates outward. In Fig. 3, the forced innermost disturbance does not decrease, and the VRW diagonally propagates outward, resulting in the formation of a spiral-shaped distribution of disturbance vorticity.

\section{Exponentially growing solution}

In this section, we consider the case in which the basic vortex has at least one moat. For $N=2$ and $\eta=$ $\frac{\bar{q}_{2}}{\bar{q}_{1}}<0$, the discriminant $D$ in (4.3) may be negative so that the eigenvalues $\lambda_{1,2}$ in (4.3) may be complex conjugate to each other. If the instability criterion

$$
\begin{aligned}
& \eta_{c}^{-}<\eta<\eta_{c}^{+} \quad \text { where } \\
& \eta_{c}^{\mp}=-\left(\sqrt{|m|-|m| \varepsilon^{2}-1+\varepsilon^{2|m|}} \pm \varepsilon^{|m|}\right)^{2}
\end{aligned}
$$

is satisfied, the eigenvalues $\lambda_{1,2}$ become complex conjugate, and the solution grows exponentially in time. In this case, the free solution (3.3) becomes the following two-component equation:

$$
\begin{aligned}
& {\left[\begin{array}{l}
r_{1} q_{1}(t, \theta) \\
r_{2} q_{2}(t, \theta)
\end{array}\right]=} \\
& 2 \cosh m \lambda_{I} t \cdot \cos m\left(\theta-\lambda_{R} t\right) \cdot\left[\frac{|R\rangle\langle L|}{\langle L \mid R\rangle}\right]_{R}|\tilde{q}(0)\rangle \\
& -2 \sinh m \lambda_{I} t \cdot \sin m\left(\theta-\lambda_{R} t\right) \cdot\left[\frac{|R\rangle\langle L|}{\langle L \mid R\rangle}\right]_{I}|\tilde{q}(0)\rangle .
\end{aligned}
$$

When the instability criterion (5.1) is not satisfied, the free solution becomes stable, which is given by the first term on the RHS of (4.5).

For $|m|=1$, the instability criterion (5.1) becomes $-\varepsilon^{2}<\eta<-\varepsilon^{2}$, which cannot be satisfied. Indeed, the discriminant in (4.3) becomes $D=(\varepsilon+\eta)^{2} \geq 0$, implying the reality of $\lambda_{1,2}$. Therefore, for $N=2$ and $|m|=1$, the solution cannot grow exponentially.

For $|m|=2$, the instability criterion (5.1) becomes $-1<\eta<-\left(1-2 \varepsilon^{2}\right)^{2}$, where $\eta=\frac{\bar{q}_{2}}{\bar{q}_{1}}<0$ and $\varepsilon=\frac{r_{1}}{r_{2}}<$ 1. This implies that the solution grows exponentially only if $\bar{q}_{1}>0, \bar{q}_{2}<0$, and $\bar{q}_{1}>\left|\bar{q}_{2}\right|$, i.e., only if the central positive vorticity $\bar{q}_{1}-\left|\bar{q}_{2}\right|$ for $0 \leq r<r_{1}$ is surrounded by the negative vorticity $-\left|\bar{q}_{2}\right|$ for $r_{1}<r<$ $r_{2}$. This is consistent with the results of Flierl (1988), Schubert et al. (1999), and Terwey and Montgomery (2002). They showed that disturbances with $m=2$ cannot grow exponentially in the usual three-region model with an inner region with positive low vorticity, an annulus with high vorticity, and an environment with zero vorticity. The exponentially growing solution (5.2) for $N=2$ and $|m|=2$ starting from an initial value at the inner radius $r_{1}$ shows both azimuthal and radial propagation and interaction between disturbances at $r_{1}$ and $r_{2}$.

Different from the case of the usual three-region model, disturbance with $m=2$ can grow exponentially in the $[N+1(\geq 4)]$ region model. To clearly see radial propagation, the exponentially growing solution (3.3) for $N=5$ and $|m|=2$ of the basic vortex $\bar{q}$ that has a central moat for $r<r_{1}$ (i.e., $\bar{q}_{1}<0, \bar{q}_{j}>0$ for $j \geq 2$ ) to mimic the annular ring of eyewall vorticity (see Fig. $4)$ is shown in Fig. 5.

The solution starting from the innermost radius $r_{1}$ shows the outward propagation of VRWs, which generates the disturbances at outer radii $r_{j}, j \geq 2$. The 

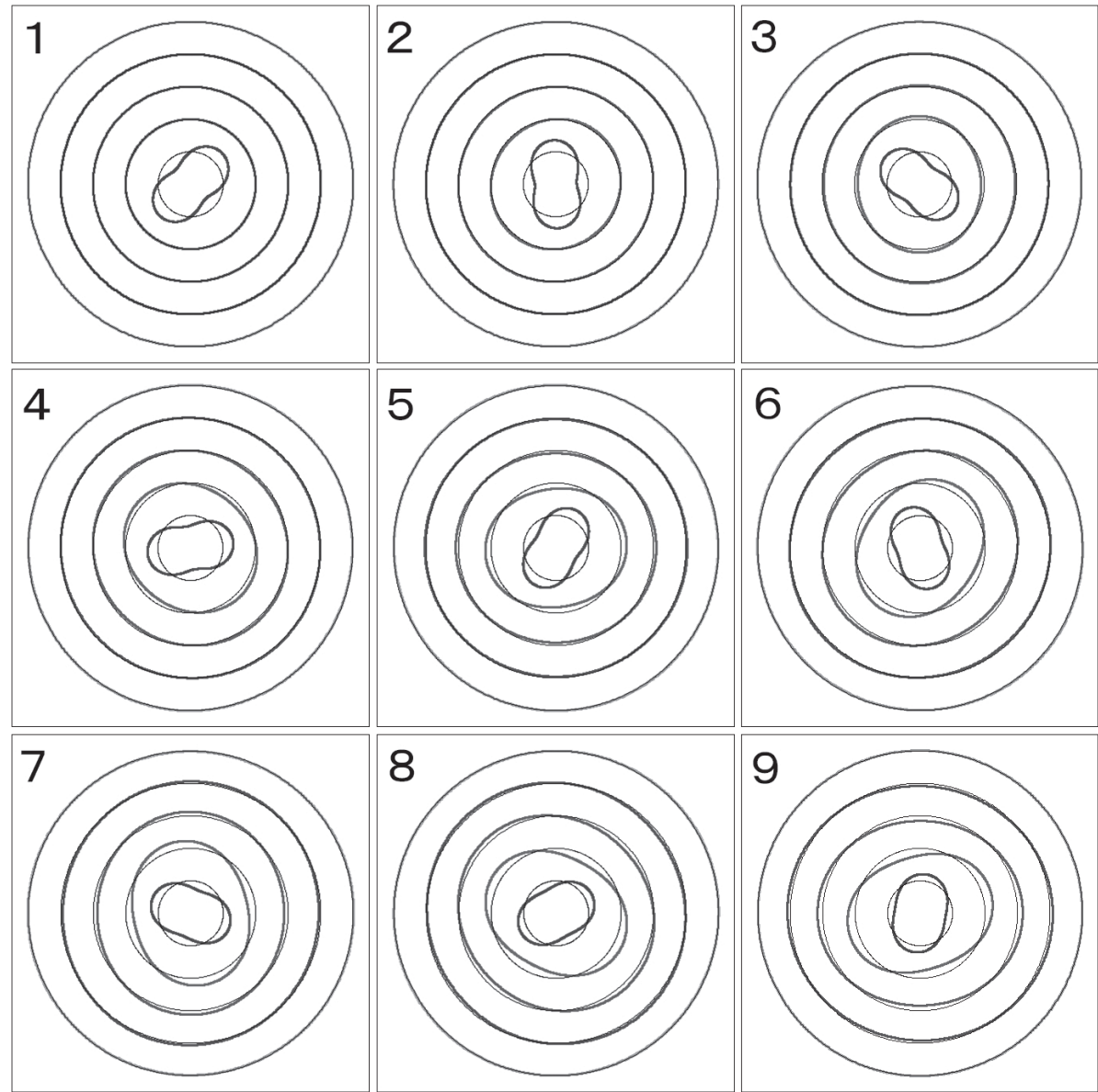

Fig. 2. Temporal evolution of a stable solution with $|m|=2$ and $N=5$. The solution starts from the innermost initial value.

VRWs at $r_{1}$ and $r_{2}$ propagate azimuthally counter to each other, since the radial gradients of $\bar{q}$ are opposite to each other there. Because of the counterpropagation (the Rayleigh condition), and because of the additional instability condition on $\bar{q}$ (the Fjørtoft condition), the VRWs at $r_{1}$ and $r_{2}$ take an upshear-tilted phase-locked structure to grow exponentially in time (see Fig. 6).

The circulation caused by the growing VRWs at $r_{1}$ and $r_{2}$ generates the disturbances also at outer radii $r_{j}$, $j \geq 3$. As a result, the whole solution grows exponentially in time. If the additional instability condition on $\bar{q}$ (the Fjørtoft condition) is not satisfied, the solution becomes stable. The marginal solution between the stable and exponentially unstable solutions grows linearly in time (not shown).

\section{Algebraically growing solution}

In this section, we consider the growing solution as a linear function of time, as a result of the degeneracy of two eigenvalues into one. Thereafter, we compare our results for $m=1$ with those of Nolan and Montgomery (2000).

\subsection{Linearly growing solution}

In the case of $N=2$, the eigenvalues $\lambda_{1,2}$ (4.3) degenerate into one if the discriminant $D$ vanishes. From (5.1), this occurs as $\eta \rightarrow \eta_{c}=\eta_{c}^{+}$or $\eta_{c}^{-}$, and then $\lambda_{1,2} \rightarrow \lambda_{c}=\frac{\bar{q}_{1}}{4}\left(1+2 \eta_{c}+\varepsilon^{2}-\frac{1+\eta_{c}}{|m|}\right)$. From (4.4), the scalar product of eigenvectors can be written in terms of $D$ as

$$
\left\langle R_{1,2} \mid L_{1,2}\right\rangle=\frac{D}{2} \mp\left(1-\varepsilon^{2}-\frac{1}{|m|}+\frac{\eta}{|m|}\right) \frac{\sqrt{D}}{2},
$$

which also vanishes as $\eta \rightarrow \eta_{c}$. By using the completeness relation $\sum_{n=1}^{2} \frac{\left|R_{n}\right\rangle\left\langle L_{n}\right|}{\left\langle L_{n} \mid R_{n}\right\rangle}=1$, the free solution, i.e., the first term on the RHS of (3.2) with $N=2$, 

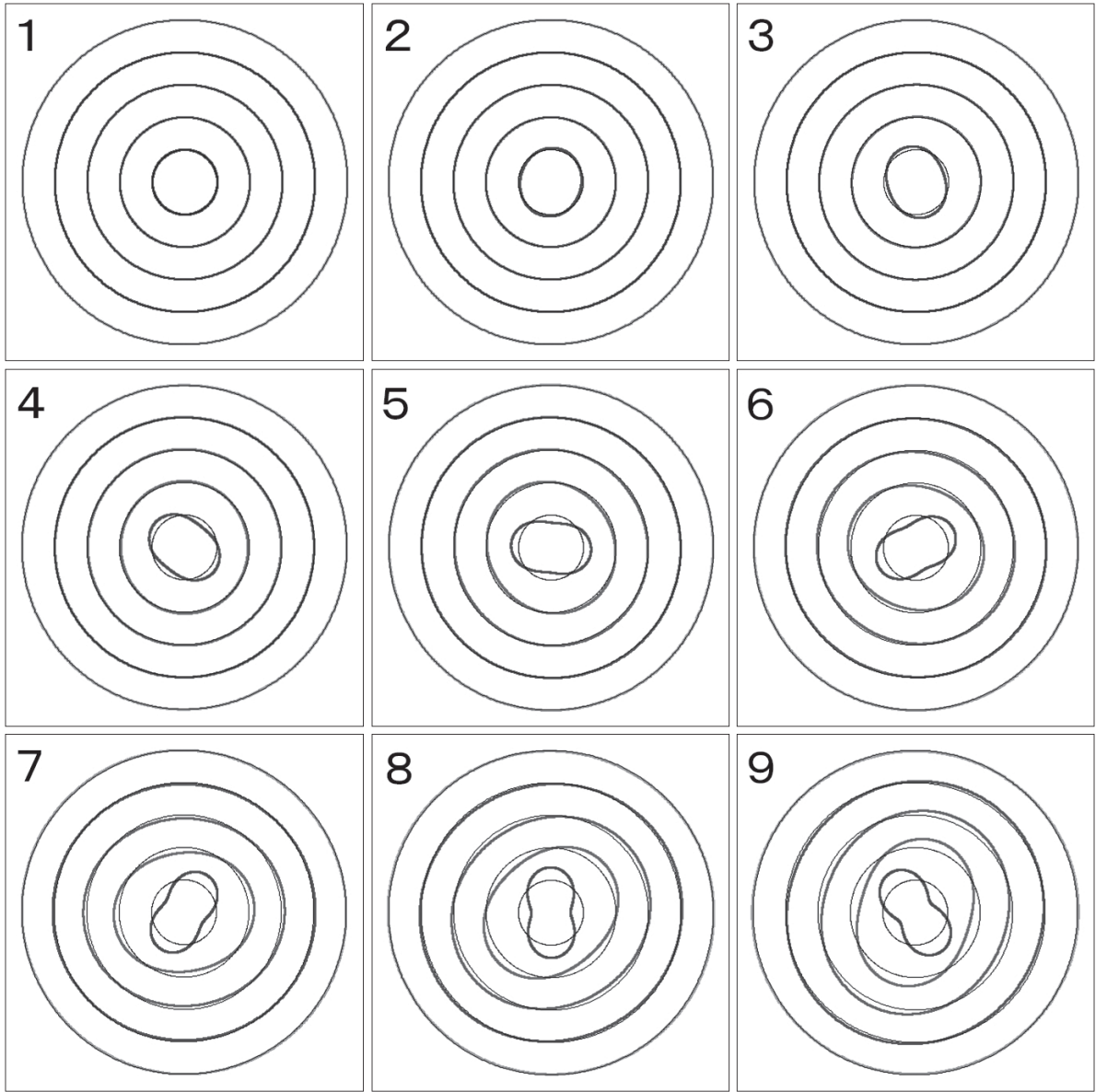

Fig. 3. Temporal evolution of a forced solution for $|m|=2$ and $N=5$. The solution starts from a null initial value and is forced at the innermost radius.

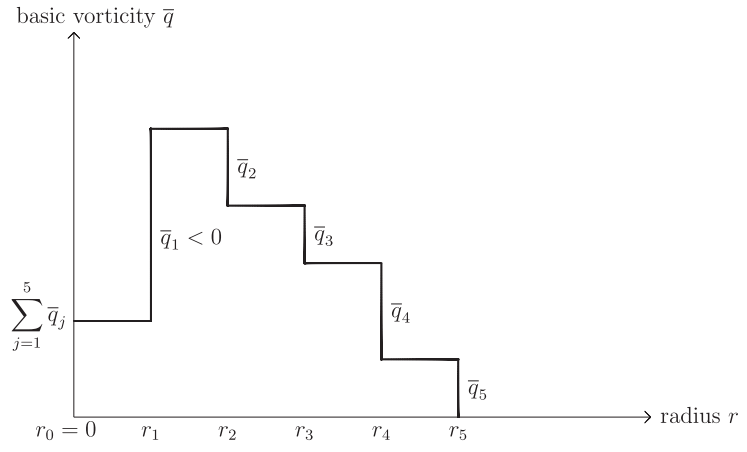

Fig. 4. Radial distribution of basic vorticity with a central moat for $N=5$. can be rewritten as

$$
\begin{aligned}
& {\left[\begin{array}{l}
r_{1} q_{1}(t, \theta) \\
r_{2} q_{2}(t, \theta)
\end{array}\right]=} \\
& \frac{\cos m\left(\theta-\lambda_{1} t\right)-\cos m\left(\theta-\lambda_{2} t\right)}{\left\langle L_{1} \mid R_{1}\right\rangle}\left|R_{1}\right\rangle\left\langle L_{1} \mid \tilde{q}(0)\right\rangle \\
& +\cos m\left(\theta-\lambda_{2} t\right) \cdot|\tilde{q}(0)\rangle .
\end{aligned}
$$

By the substitution of (6.1) and $\lambda_{2}-\lambda_{1}=-\frac{\bar{q}_{1}}{2} \sqrt{D}$, and taking the limit of $D \rightarrow 0$, the solution (6.2) grows as a linear function of time:

$$
\begin{aligned}
& {\left[\begin{array}{l}
r_{1} q_{1}(\theta, t) \\
r_{2} q_{2}(\theta, t)
\end{array}\right]=} \\
& -\frac{m|m| \bar{q}_{1} t}{|m|-|m| \varepsilon^{2}-1+\eta_{c}} \sin m\left(\theta-\lambda_{c} t\right) \cdot\left|R_{c}\right\rangle\left\langle L_{c} \mid \tilde{q}(0)\right\rangle \\
& +\cos m\left(\theta-\lambda_{c} t\right) \cdot|\tilde{q}(0)\rangle \text { for } \eta=\eta_{c} .
\end{aligned}
$$



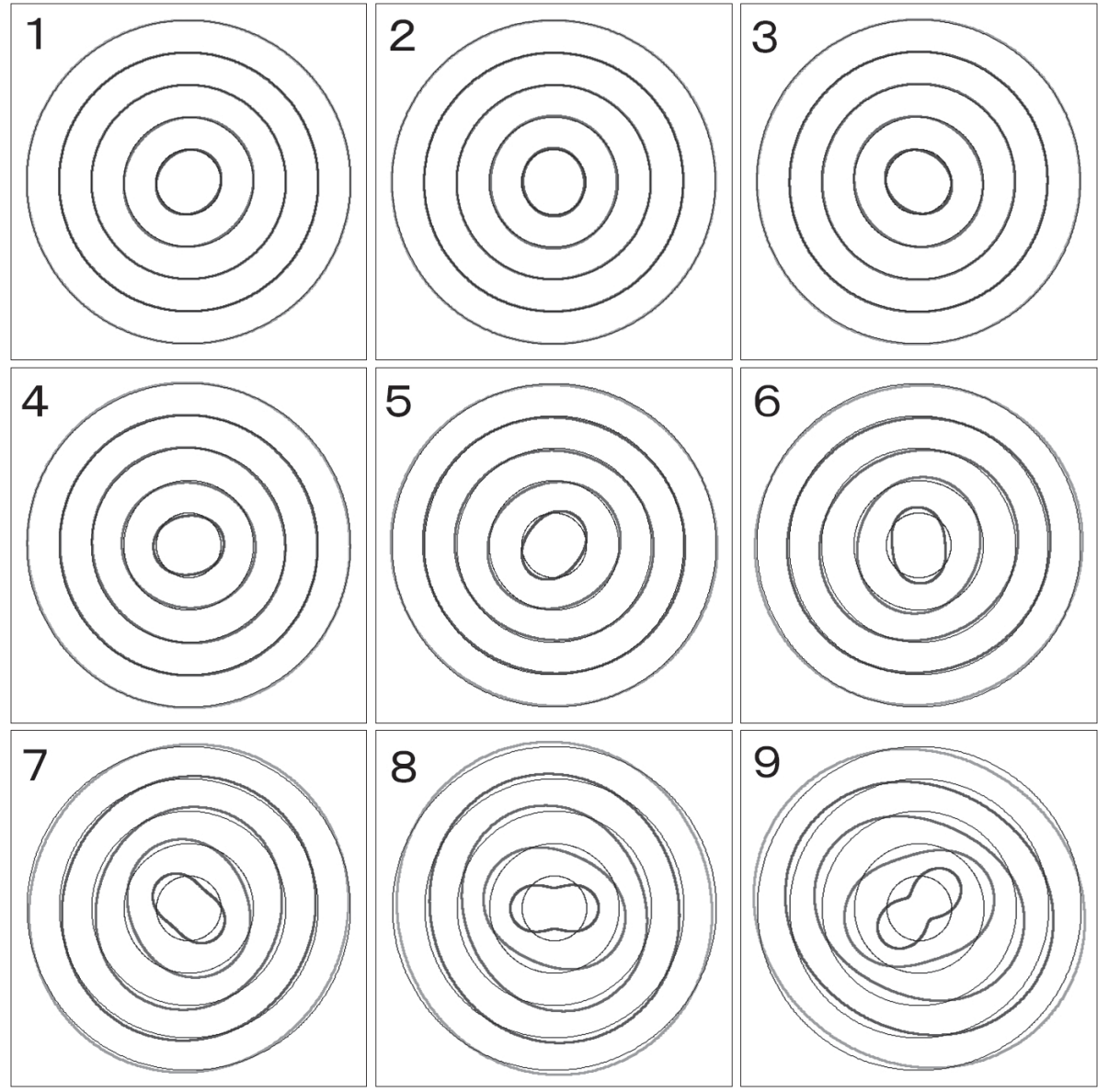

Fig. 5. Temporal evolution of an exponentially growing solution for $|m|=2$ and $N=5$. The solution starts from the innermost initial value.

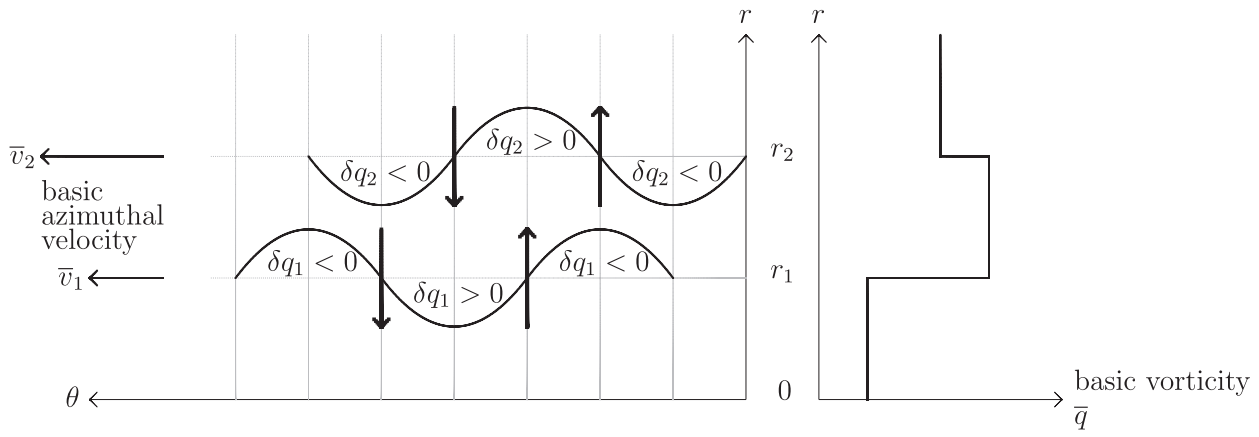

Fig. 6. Upshear-tilted phase-locked structure. The curves are the boundaries between the uniform vorticity regions. The vertical arrows represent the circulations induced by the vorticity disturbances $\delta q$. The circulation induced by $\delta q_{1}$ amplifies $\delta q_{2}$ and vice versa. 
Here $\left|R_{c}\right\rangle\left\langle L_{c}\left|=\lim _{\eta \rightarrow \eta_{c}}\right| R_{1,2}\right\rangle\left\langle L_{1,2}\right|$.

The linearly growing solution (6.3) is the limiting case of the stable free solution [the first term on the RHS of (4.5)] as $\eta \rightarrow \eta_{c}$, and at the same time, the limiting case of the exponentially growing free solution (5.2) as $\eta \rightarrow \eta_{c}$ from the opposite side. In other words, $(6.3)$ is the marginal solution between the stable and exponentially unstable solutions.

In particular, the linearly growing solution (6.3) also exists for $|m|=1$, although there cannot exist any exponentially growing solution for $|m|=1$. For $|m|=$ 1 , the eigenvalues (4.3) become

$$
\lambda_{1,2}=\frac{\bar{q}_{1}}{4}\left(\varepsilon^{2}+\eta \pm \sqrt{D}\right), \quad D=\left(\varepsilon^{2}+\eta\right)^{2} \geq 0 .
$$

As $\eta \rightarrow \eta_{c}=-\varepsilon^{2}$, i.e., as $D \rightarrow 0$, the eigenvalues $\lambda_{1}$ and $\lambda_{2}$ degenerate into the marginal eigenvalues $\lambda_{c}=$ 0 . As a result of this degeneracy, the free solution grows linearly, as is given by (6.3) with $|m|=1$.

The linear growth for $|m|=1$ in spite of the absence of exponential growth also occurs in the case of $N \geq$ 3 , as shown below. As is already known, the Fourier transform of the free linearized vorticity equation (2.1) with $\hat{\dot{q}}(t, r)=0$ has the following exact solution for $|m|=1$ (Smith and Rosenbluth 1990):

$$
\begin{aligned}
\hat{q}(t, r)= & e^{-i \bar{\omega}(r) t} \hat{q}(0, r) \\
& -i t \frac{d \bar{q}}{d r} \int_{r}^{\infty} d \xi e^{-i \bar{\omega}(\xi) t} \frac{1}{\xi^{3}} \int_{0}^{\xi} d \eta \eta^{2} \hat{q}(0, \eta) .
\end{aligned}
$$

The presence of $t$ in the second term on the RHS of (6.5) does not directly imply linear growth but it represents the shearing effect of differential rotation. Substituting (2.5), (2.7), and (2.9) into (6.5) and partially integrating gives the following discrete solution:

$$
\begin{aligned}
& \hat{q}_{j}(t)=e^{-i \bar{\omega}_{j} t} \hat{q}_{j}(0) \\
& -\sum_{k=j}^{N} \frac{\left(r_{k+1}^{2}-r_{k}^{2}\right) \bar{q}_{j}}{2 r_{k+1}^{2} r_{k}^{2}\left(\bar{\omega}_{k+1}-\bar{\omega}_{k}\right)}\left(e^{-i \bar{\omega}_{k+1} t}-e^{-i \bar{\omega}_{k} t}\right) \sum_{l=1}^{k} r_{l}^{2} \hat{q}_{l}(0), \\
& j=1,2, \ldots, N,
\end{aligned}
$$

where $\bar{\omega}_{N+1}=0$ and $r_{N+1}=\infty$. Since $\bar{\omega}_{2}-\bar{\omega}_{1}=$ $\frac{\bar{q}_{1}}{2}\left\{\left(\frac{r_{1}}{r_{2}}\right)^{2}-1\right\}$ from (2.9), the discrete solution (6.6) can be rewritten as follows:

$$
\begin{aligned}
& \hat{q}_{1}(t)=e^{-i \bar{\omega}_{2} t} \hat{q}_{1}(0) \\
& -\sum_{k=2}^{N} \frac{\left(r_{k+1}^{2}-r_{k}^{2}\right) \bar{q}_{1}}{2 r_{k+1}^{2} r_{k}^{2}\left(\bar{\omega}_{k+1}-\bar{\omega}_{k}\right)}\left(e^{-i \bar{\omega}_{k+1} t}-e^{-i \bar{\omega}_{k t}}\right) \sum_{l=1}^{k} r_{l}^{2} \hat{q}_{l}(0), \\
& \hat{q}_{j}(t)=e^{-i \bar{\omega}_{j}^{t}} \hat{q}_{j}(0) \\
& -\sum_{k=j}^{N} \frac{\left(r_{k+1}^{2}-r_{k}^{2}\right) \bar{q}_{j}}{2 r_{k+1}^{2} r_{k}^{2}\left(\bar{\omega}_{k+1}-\bar{\omega}_{k}\right)}\left(e^{-i \bar{\omega}_{k+1} t}-e^{-i \bar{\omega}_{k} t}\right) \sum_{l=1}^{k} r_{l}^{2} \hat{q}_{l}(0) \\
& \text { for } \quad 2 \leq j \leq N .
\end{aligned}
$$

Comparing (6.7) with the general form of the free solution, i.e., the first term on the RHS of (3.1) with $|m|=1$, we can see that the set of eigenvalues of $\Lambda$ is equal to the set of basic angular velocities. Without loss of generality, we can say that

$$
\lambda_{n}=\bar{\omega}_{n+1} \quad \text { for }|m|=1, \quad n=1,2, \ldots, N .
$$

As is easily seen, (6.4) is a particular case of (6.8) for $N=2$ since $\bar{\omega}_{2}=\frac{\bar{q}_{1}}{2}\left(\frac{r_{1}}{r_{2}}\right)^{2}+\frac{\bar{q}_{2}}{2}=\frac{\bar{q}_{1}}{2}\left(\varepsilon^{2}+\eta\right)$ and $\bar{\omega}_{3}=0$ for $N=2$. When $\bar{\omega}_{M+1}=\bar{\omega}_{M}$ for some $M(2 \leq M \leq N)$, i.e., when $\lambda_{M}=\lambda_{M-1}$ for some $M(2 \leq M \leq N)$, the discrete solution (6.7) grows linearly in time:

$$
\begin{aligned}
& \hat{q}_{1}(t)=e^{-i \bar{\omega}_{2} t} \hat{q}_{1}(0) \\
& -\sum_{k=2(k \neq M)}^{N} \frac{\left(r_{k+1}^{2}-r_{k}^{2}\right) \bar{q}_{1}}{2 r_{k+1}^{2} r_{k}^{2}\left(\bar{\omega}_{k+1}-\bar{\omega}_{k}\right)}\left(e^{-i \bar{\omega}_{k+1} t}-e^{-i \bar{\omega}_{k} t}\right) \sum_{l=1}^{k} r_{l}^{2} \hat{q}_{l}(0)
\end{aligned}
$$

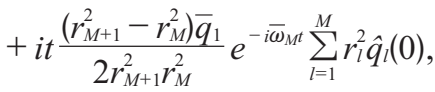

$$
\begin{aligned}
& \hat{q}_{j}(t)=e^{-i \bar{\omega}_{j} t} \hat{q}_{j}(0) \\
& -\sum_{k=j(k \neq M)}^{N} \frac{\left(r_{k+1}^{2}-r_{k}^{2}\right) \bar{q}_{j}}{2 r_{k+1}^{2} r_{k}^{2}\left(\bar{\omega}_{k+1}-\bar{\omega}_{k}\right)}\left(e^{-i \bar{\omega}_{k+1} t}-e^{-i \bar{\omega}_{k} t}\right) \sum_{l=1}^{k} r_{l}^{2} \hat{q}_{l}(0) \\
& +i t \frac{\left(r_{M+1}^{2}-r_{M}^{2}\right) \bar{q}_{j}}{2 r_{M+1}^{2} r_{M}^{2}} e^{-i \bar{\omega}_{M} \sum_{l=1}^{M} r_{l}^{2} \hat{q}_{l}(0)} \quad \text { for } \quad 2 \leq j \leq M \text {, } \\
& \hat{q}_{j}(t)=e^{-i \bar{\omega}_{j} t} \hat{q}_{j}(0) \\
& -\sum_{k=j}^{N} \frac{\left(r_{k+1}^{2}-r_{k}^{2}\right) \bar{q}_{j}}{2 r_{k+1}^{2} r_{k}^{2}\left(\bar{\omega}_{k+1}-\bar{\omega}_{k}\right)}\left(e^{-i \bar{\omega}_{k+1} t}-e^{-i \bar{\omega}_{k} t}\right) \sum_{l=1}^{k} r_{l}^{2} \hat{q}_{l}(0) \\
& \text { for } M+1 \leq j \leq N \text {. }
\end{aligned}
$$

$\bar{\omega}_{M+1}=\bar{\omega}_{M}$ implies $\sum_{j=1}^{M} \bar{q}_{j} r_{j}^{2}=0$ from (2.9). This means that the Rayleigh condition is satisfied, i.e., at least one $\bar{q}_{j}<0$ for some $j(1 \leq j \leq M)$. Interestingly, linear growth occurs only inside $r_{M}$. Formally, the linear growth in (6.9) may be regarded as the result of the resonance between two internal modes $e^{-i \lambda_{M} t}$ and $e^{-i \lambda_{M-1} t}$ with $\lambda_{M}=\lambda_{M-1}$.

As an example, after the inverse Fourier transformation of (6.9), the case of $N=5$ and $M=2$ is shown in Fig. 7. To mimic the annular ring of eyewall vorticity, the cases of $\bar{q}_{1}<0$ and $\bar{q}_{j}>0,2 \leq j \leq 5$ (see Fig. 5) are shown in Fig. 7.

The solution starting from the innermost radius $r_{1}$ shows the outward propagation of VRWs, which generates the disturbances at outer radii $r_{j}, j \geq 2$. The VRWs at $r_{1}$ and $r_{2}$ propagate azimuthally counter to each other, since the radial gradients of $\bar{q}$ are opposite to each other there. However, different from the exponentially growing solution in Fig. 4, the solution does not take an upshear-tilted phase-locked structure. Instead, the solution asymptotically takes an antiphase structure between $r_{1}$ and $r_{2}$ as $t \rightarrow \infty$. Because of this asymptotic antiphase structure, the solution grows linearly in time instead of exponentially. Further, 

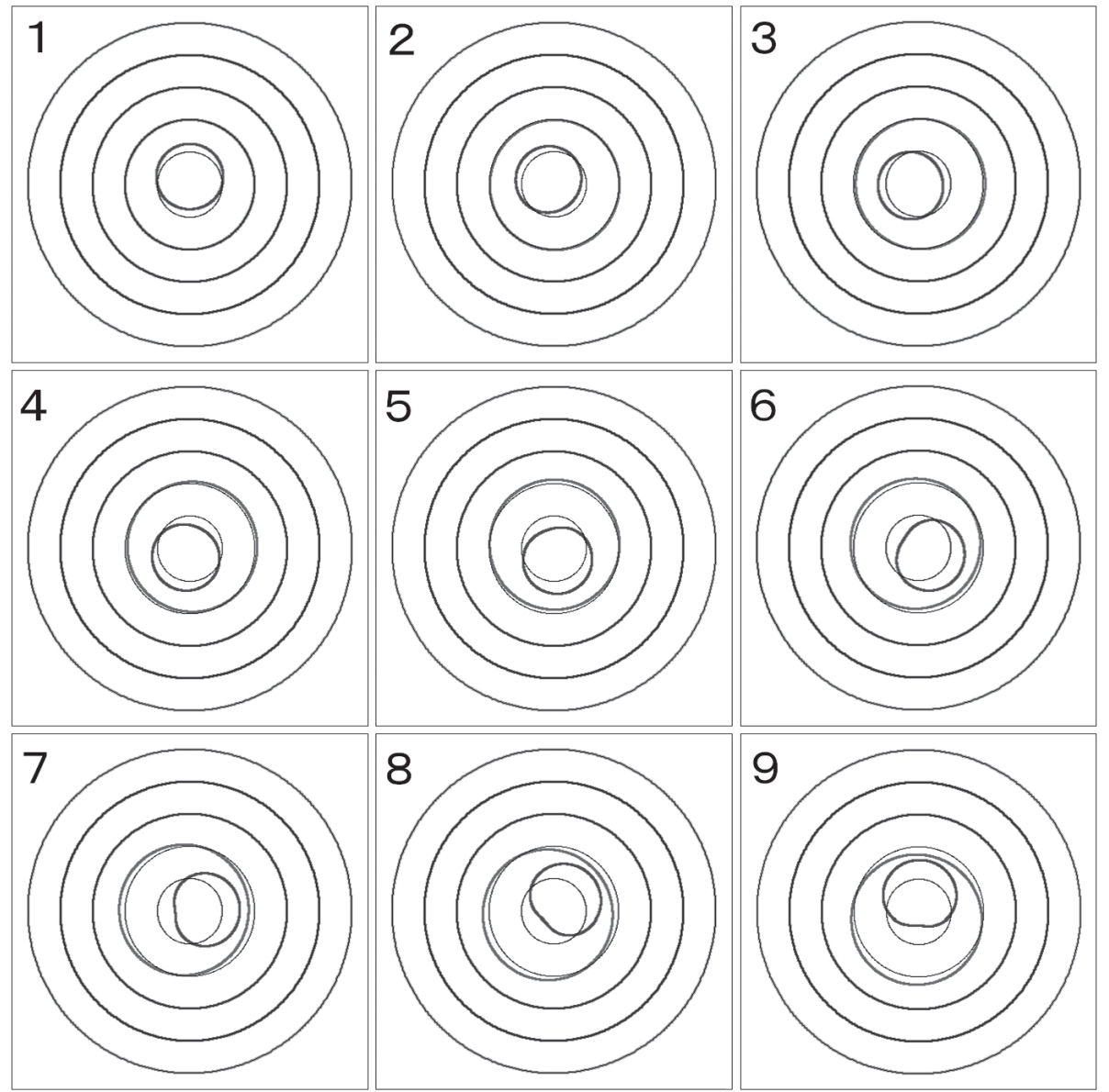

Fig. 7. Temporal evolution of a linearly growing solution for $|m|=1$ and $N=5$. The solution starts from the innermost initial value.

because of this antiphase structure, the circulations caused by the disturbances at $r_{1}$ and $r_{2}$ significantly cancel each other, resulting in the confinement of growth inside $r_{2}$.

The difference between the exponential growth of $m=2$ in Fig. 4 and the linear growth of $m=1$ in Fig. 7 is clearly seen in the Hovmoeller diagrams (see Fig. 8).

The growth of $m=2$ (Fig. 8a) is rapid compared with that of $m=1$ (Fig. 8b).

\subsection{Comparison with Nolan and Montgomery (2000)}

To compare the discrete solution (6.9) with the continuous solution (2.16) of Nolan and Montgomery (2000) (NM hereafter), the solution (6.9) is rewritten as follows:

$$
\begin{aligned}
& \hat{q}_{j}(t)=i t H_{M j} \bar{q}_{j} \frac{\left(r_{M+1}^{2}-r_{M}^{2}\right)}{2 r_{M+1}^{2} r_{M}^{2}} e^{-i \bar{\omega}_{M t}} \sum_{l=1}^{M} r_{l}^{2} \hat{q}_{l}(0)+\frac{\bar{q}_{j}}{2 r_{N}^{2} \bar{\omega}_{N}} \sum_{l=1}^{N} r_{l}^{2} \hat{q}_{l}(0)-\bar{q}_{j} \sum_{k=j(k+1, M)}^{N-1} \frac{\left(r_{k+1}^{2}-r_{k}^{2}\right)}{2 r_{k+1}^{2} r_{k}^{2}\left(\bar{\omega}_{k+1}-\bar{\omega}_{k}\right)}\left(e^{-i \bar{\omega}_{k+1} t}-e^{-i \bar{\omega}_{k} t}\right) \sum_{l=1}^{k} r_{l}^{2} \hat{q}_{l}(0) \\
& -\frac{\bar{q}_{j}}{2 r_{N}^{2} \bar{\omega}_{N}} e^{-i \bar{\omega}_{N} t} \sum_{l=1}^{N} r_{l}^{2} \hat{q}_{l}(0)+H_{j 2} e^{-i \bar{\omega}_{j} t} \hat{q}_{j}(0)+\delta_{j 1} e^{-i \bar{\omega}_{2} t} \hat{q}_{1}(0) \quad \text { for } \quad 1 \leq j \leq N,
\end{aligned}
$$

where $H_{M j}=1$ if $M \geq j$ and $H_{M j}=0$ if $M<j$.

The first term on the RHS of (6.10) represents algebraic growth, which is proportional to $t$. Although algebraic growth represented by the first term of (2.16) of NM is proportional to $\sqrt{t}$ instead of $t$, there are qualitative correspondences between the discrete (6.10) and the continuous (2.16) of NM.

- Radial dependence: The growing part is present 
(a)

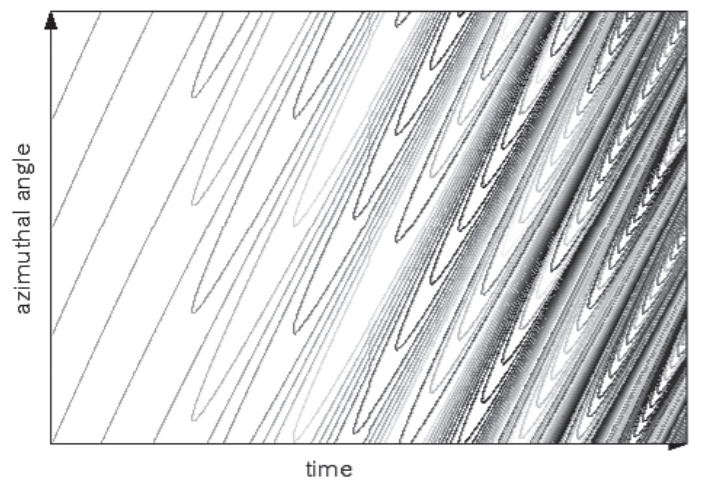

(b)

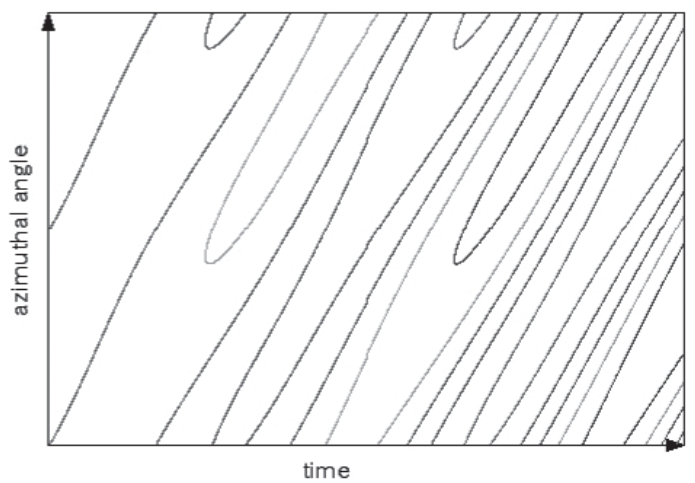

Fig. 8. Comparison of the exponential growth of $m=2$ (a) and the linear growth of $m=1$ (b) at $r=r_{1}$. All conditions are the same both (a) and (b) except for the wavenumbers. The contours represent isolines of the amplitude of disturbance.

only inside the radius of maximum angular velocity, and there it depends on the radial distance only through the gradient of the basic vorticity. That is, it is proportional to $H_{M j} \bar{q}_{j}$ in (6.10) and proportional to $H\left(r_{\max }-r\right) \frac{d \bar{q}(r)}{d r}$ in $(2.16)$ of NM, where $H(r)$ is the Heaviside step function, and $r_{\max }$ is the radius of maximum angular velocity.

- Temporal dependence: The growing part rotates with the maximum angular velocity. That is, it is proportional to $t e^{-i \bar{\omega}_{M t} t}$ in (6.10) and proportional to $\sqrt{t} e^{-i \bar{\omega}\left(r_{\text {max }}\right) t}$ in $(2.16)$ of NM.

- Dependence on initial conditions: The growing part is absent if the initial disturbance is absent inside the radius of maximum angular velocity. That is, it is proportional to $\sum_{l=1}^{M} r_{l}^{2} \hat{q}_{l}(0)$ in (6.10) and proportional to $\int_{0}^{r_{\max }} d r r^{2} \hat{q}(0, r)$ in (2.16) of NM.

The second term on the RHS of (6.10) represents the steady pseudomode. This term corresponds to the second term of (2.16) of NM with the outermost radius
$R \rightarrow \infty$. The steady pseudomode depends on the radial distance only through the radial gradient of the basic vorticity. That is, it is proportional to $\bar{q}_{j}$ in (6.10) and proportional to $\frac{d \bar{q}(r)}{d r}$ in (2.16) of NM. This radial dependence implies that the steady pseudomode is generated by the linear displacement of the vortex.

The third term on the RHS of (6.10) represents the sheared VRWs as indicated by the factor $\left(e^{-i \bar{\omega}_{k+1} t}-\right.$ $\left.e^{-i \bar{\omega}_{k} t}\right)$. This term corresponds to the third term of (2.16) of NM, which is also interpreted as the sheared VRWs.

The other terms on the RHS of (6.10) are required to satisfy the initial conditions.

\subsection{Algebraic growth proportional to tinstead of $\sqrt{t}$}

The algebraic growth in (6.10) is proportional to $t$ instead of $\sqrt{t}$. The difference can be explained as follows. On the assumption that $\bar{\omega}(r)$ is maximum at a radius $r=r_{\max }$, i.e., $\bar{\omega}^{\prime}\left(r_{\max }\right)=\left[\frac{d \bar{\omega}(r)}{d r}\right]_{r=r_{\text {max }}}=0$ and $\bar{\omega}^{\prime \prime}\left(r_{\max }\right)$ $=\left[\frac{d^{2} \bar{\omega}(r)}{d r^{2}}\right]_{r=r_{r .0}}<0, \mathrm{NM}$ asymptotically (i.e., for large $\left.t\right)$ estimated the second term of (6.5) as

$$
\begin{aligned}
-i t \frac{d \bar{q}(r)}{d r} \int_{r}^{\infty} d \xi h(\xi) e^{-i \bar{\omega}(\xi) t} & =-i t \frac{d \bar{q}(r)}{d r} \int_{r}^{\infty} d \xi h(\xi) e^{-i \bar{\omega}\left(r_{\text {max }}\right) t-i \frac{1}{2} \bar{\omega}^{\prime \prime}\left(r_{\text {max }}\right)\left(\xi-r_{\text {max }}\right)^{2}+\cdots} \\
& \approx-i t \frac{d \bar{q}(r)}{d r} h\left(r_{\text {max }}\right) e^{-i \bar{\omega}\left(r_{\text {max }}\right) t} H\left(r_{\text {max }}-r\right) \int_{-\infty}^{+\infty} d \xi e^{-i \frac{1}{2} \bar{\omega}^{\prime \prime}\left(r_{\text {max }}\right)\left(\xi-r_{\text {max }}\right)^{2} t} \\
& =-i t \frac{d \bar{q}(r)}{d r} h\left(r_{\text {max }}\right) e^{-i \bar{\omega}\left(r_{\text {max }}\right) t} H\left(r_{\text {max }}-r\right) \sqrt{\frac{2}{t \mid \bar{\omega}^{\prime \prime}\left(r_{\text {max }}\right)}} \int_{-\infty}^{+\infty} d \eta e^{i \eta^{2}} \\
& \propto \sqrt{\frac{t}{\mid \bar{\omega}^{\prime \prime}\left(r_{\text {max }}\right)}} \frac{d \bar{q}(r)}{d r} h\left(r_{\text {max }}\right) e^{-i \bar{\omega}\left(r_{\text {max }}\right) t} H\left(r_{\text {max }}-r\right),
\end{aligned}
$$

where $h(\xi)=\frac{1}{\xi^{3}} \int_{0}^{\xi} d \eta \eta^{2} \hat{q}(0, \eta)$. However, in the discrete model, the maximum of the basic angular velocity $\bar{\omega}(r)$ is formulated as $\bar{\omega}_{M}=\bar{\omega}_{M+1}$ for some $M$ (see Fig. 9).

We suppose that discrete formulation may corre- 

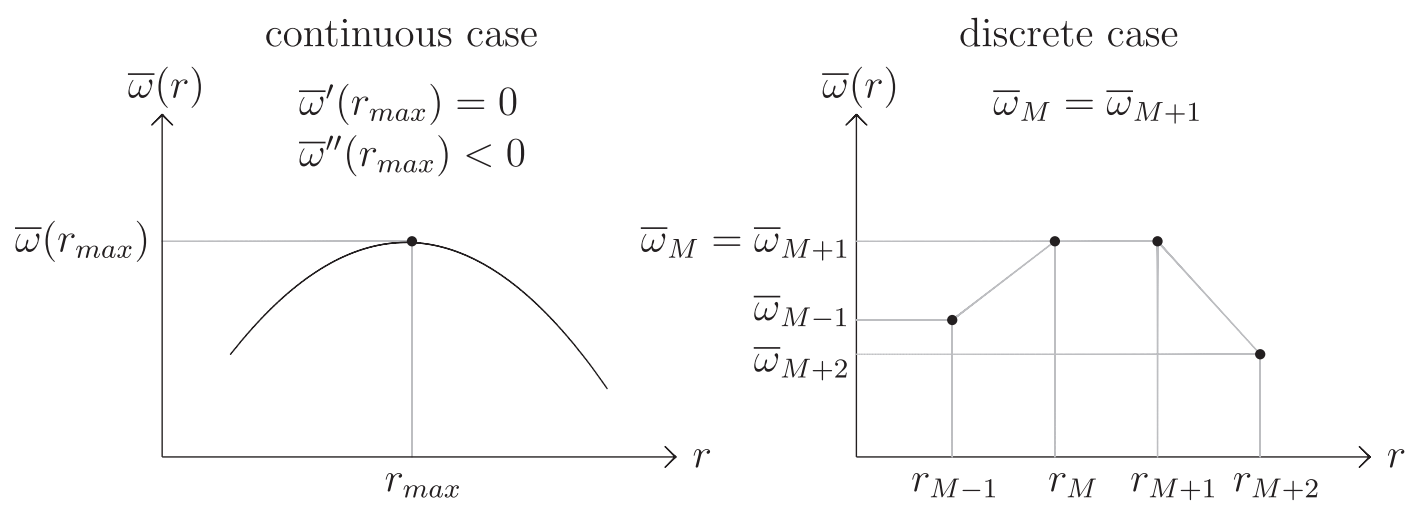

Fig. 9. Maximum angular velocity for the continuous and discrete cases.

spond to a continuous case of

$$
\begin{gathered}
\bar{\omega}^{\prime}\left(r_{\max }\right)=\bar{\omega}^{\prime \prime}\left(r_{\max }\right)=\cdots=\left[\frac{d^{2 \nu-1} \bar{\omega}(r)}{d r^{2 \nu-1}}\right]_{r=r_{\max }}=0 \\
\text { and }\left[\frac{d^{2 \nu} \bar{\omega}(r)}{d r^{2 \nu}}\right]_{r=r_{\max }}=\bar{\omega}^{(2 \nu)}\left(r_{\max }\right)<0
\end{gathered}
$$

with some large integer $\nu$. In this case, asymptotic estimation becomes

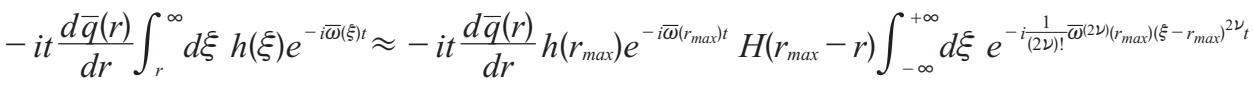

$$
\begin{aligned}
& =-i t \frac{d \bar{q}(r)}{d r} h\left(r_{\max }\right) e^{-i \bar{\omega}\left(r_{\max }\right) t} H\left(r_{\max }-r\right)\left\{\frac{(2 \nu) !}{t\left|\bar{\omega}^{(2 \nu)}\right|}\right\}^{\frac{1}{2 \nu}} \int_{-\infty}^{+\infty} d \eta e^{i \eta^{2 \nu}} \\
& \propto t^{1-\frac{1}{2 \nu}} \frac{d \bar{q}(r)}{d r} h\left(r_{\max }\right) e^{-i \bar{\omega}\left(r_{\max }\right) t} H\left(r_{\max }-r\right) .
\end{aligned}
$$

This results in linear growth in time in the limit of $\nu \rightarrow$ $\infty$.

\subsection{Growth as a result of resonance}

The linear growth in time in (6.10) is the result of the coincidence of the two adjacent angular velocities $\bar{\omega}_{M}$ and $\bar{\omega}_{M+1}$, which means the degeneracy of two eigenvalues $\lambda_{M-1}$ and $\lambda_{M}$. The linear growth caused by the degeneracy of two eigenvalues is well known. For example, let us consider the following initial value problem:

$$
\left(\frac{d}{d t}-i a\right)\left(\frac{d}{d t}-i b\right) f(t)=0 \quad \text { with } \quad f(0)
$$

and $\left[\frac{d f(t)}{d t}\right]_{t=0}=\dot{f}(0) \quad$ prescribed.

The solution is given by

$$
f(t)=\frac{f(0)}{b-a}\left(b e^{i a t}-a e^{i b t}\right)+i \frac{\dot{f}(0)}{b-a}\left(e^{i a t}-e^{i b t}\right) .
$$

In the limit of $b \rightarrow a$, this becomes

$$
f(t)=f(0) e^{i a t}+t\{\dot{f}(0)-i a f(0)\} e^{i a t} .
$$

As a result of the interaction between two modes $e^{\text {iat }}$ and $e^{i b t}$ with $b \rightarrow a$, the solution grows linearly in time. This interaction may be interpreted as a resonance between $e^{i a t}$ and $e^{i b t}$. Thus, the linear growth in (6.10) may also be interpreted as the resonance between two adjacent modes $e^{-i \lambda_{M-1 t}}=e^{-i \bar{\omega}_{M t}}$ and $e^{-i \lambda_{M t}}=e^{-i \bar{\omega}_{M+1} t}$ with $\lambda_{M-1} \rightarrow \lambda_{M}$, i.e., with $\bar{\omega}_{M} \rightarrow \bar{\omega}_{M+1}$.

The interaction between $e^{-i \bar{\omega}_{M t}}$ and $e^{-i \bar{\omega}_{M+1} t}$ does not imply resonant interaction between the disturbances at $r_{M}$ and $r_{M+1}$. Rather, it is the interaction between two modes $e^{-i \lambda_{M-1} t}$ and $e^{-i \lambda_{M} t}$ inside $r_{M}$. Indeed, as shown in (6.10), the disturbance at $r_{M+1}$ does not grow.

In NM, the algebraic growth in the core region of the vortex is interpreted as follows. The sheared VRWs inside the radius of the maximum angular velocity are in the form of a leading spiral. The radial gradient of the basic vorticity is negative near the radius of the maximum angular velocity. The leading spiral in the negative radial gradient of the basic vorticity implies an inward group velocity. The inward group velocity 
results in the growth of disturbances in the core region of the vortex, in particular, near the maximum radial gradient of the basic vorticity.

Indeed, the algebraically growing term of (2.16) in NM needs the other terms to be a solution. This implies that growth is a result of the interaction between the growing term and the other terms representing sheared VRWs.

Also, in the present discrete model, the algebraically growing term of (6.10) needs the other terms to be a solution. The growing term is proportional to $t$, implying algebraic growth. At the same time, the term has no phase differences required for growth; hence, the term itself cannot be a solution. Therefore, also in the discrete case, growth can be said to be a result of the interaction between the growing term and the other terms representing sheared VRWs.

However, in the present discrete model, the radial group velocity cannot be defined properly. Because of this, the growing mechanism of NM cannot be directly applied to the discrete model, and it is not reflected in the solution (6.10). Rather, the algebraic growth in the discrete model appears to be the result of the resonance between two modes associated with the maximum angular velocity.

\section{Concluding remarks}

In this paper, we analytically solved the initial value problem of VRWs in a barotropic system on an $f$ plane, which was linearized about an axisymmetric basic flow. To analytically solve the disturbance vorticity equation, the axisymmetric basic vorticity $\bar{q}$ was assumed to be piecewise uniform in the radial direction with jumps at radii $r_{j}, 0<r_{1}<r_{2}<\cdots<r_{N}<\infty$. By the assumption of piecewise uniformity, and after the Fourier transformation in the azimuthal direction with a wavenumber $m$, the disturbance vorticity equation became a system of ordinary differential equations with respect to time; these could be analytically solved to give the formal solution of disturbance vorticity with a prescribed initial value.

The main results are summarized in Figs. 2, 3, 4, and 7. In Fig. 2, the stable solution with $m=2$ for a monopolar basic vorticity $\bar{q}$ for $N=5$ is depicted. The solution starts from the innermost radius $r_{1}$ and propagates outward.

In Fig. 3, the solution with $m=2$, which is forced at the innermost radius $r_{1}$, for the same monopolar $\bar{q}$ as in Fig. 2 is depicted. The solution starts from a null initial value and forms a spiral-shaped distribution of disturbance vorticity.

In Fig. 4, the exponentially growing solution with $m=2$ for a basic vorticity $\bar{q}$ with a central moat for $N=$ 5 is depicted. The solution starts from the innermost radius $r_{1}$ and propagates outward to form a growing spiral-shaped distribution of disturbance vorticity.

In Fig. 7, the linearly growing solution with $m=1$ for the basic vorticity $\bar{q}$ with a central moat for $N=5$ is depicted. The solution starts from the innermost radius $r_{1}$ and propagates outward but is almost confined inside $r_{2}$. The solution asymptotically takes the antiphase structure between $r_{1}$ and $r_{2}$ as $t \rightarrow \infty$.

In spite of the fact that disturbances with $m=2$ are present in some observations and numerical simulations (e.g., elliptical eye), they cannot grow in the usual three-region model with an inner region with low positive vorticity, an annulus with high positive vorticity, and an environment with zero vorticity (e.g., Flierl 1988; Schubert et al. 1999; Terwey and Montgomery 2002). Disturbances with $m=2$ can exponentially grow in a three-region model if the model has negative inner vorticity (Flierl 1988; Terwey and Montgomery 2002), negative annulus vorticity (Flierl 1988), or positive environmental vorticity (Terwey and Montgomery 2002).

The absence of exponential growth for $m=2$ in the usual three-region model, whose environmental vorticity is zero, is caused by the large vorticity jump at the outer edge of the annulus. Because the propagation velocity $C_{\text {prop }}$ of VRW is proportional to - $\frac{\text { vorticity jump }}{m}$, the large vorticity jump implies a large clockwise $C_{\text {prop }}$ at the outer edge of the annulus, which makes the phase lock with the counterclockwise propagating VRW at the inner edge of the annulus impossible. Without the phase lock between VRWs at the inner and outer edges of the annulus, exponential growth is impossible.

However, in the $[N+1(\geq 4)]$ region model having an environment with zero vorticity, disturbances with $m=2$ can grow exponentially. The environment with zero vorticity is the outermost region and is not adjacent to the annulus with high vorticity. Hence, the zero environmental vorticity does not imply a large vorticity jump at the outer edge of the annulus. Because of this, the phase lock between VRWs at the outer and inner edges of the annulus becomes possible, and as a result, the exponential growth of disturbances with $m=2$ becomes possible.

In some observations and numerical simulations, there exist growing disturbances with $m=1$ (e.g., meandering motion of the center of a hurricane). Indeed, the analytical solution for $m=1$ to the continuous vorticity equation linearized about an axisymmetric basic state (Smith and Rosenbluth 1990) shows the asymptotic algebraic growth of disturbances with 
$m=1$, if the basic angular velocity $\bar{\omega}$ has a maximum other than at the center. However, it is perceived that there exist no growing disturbances with $m=1$ in the usual three-region model, although precessional disturbances with $m=1$ are possible if the baroclinic effect is considered (Flierl 1988).

However, in the $[N+1(\geq 4)]$-region model, disturbances with $m=1$ can grow linearly in time if two adjacent basic angular velocities degenerate into one, i.e., $\bar{\omega}_{M}=\bar{\omega}_{M+1}$ for some $M(2 \leq M \leq N)$. The degeneracy corresponds to the maximum basic angular velocity $\bar{\omega}$ other than at the center in the continuous case. The algebraic growth $(\propto t)$ in the discrete case is different from that $(\propto \sqrt{t})$ in the continuous case. The difference comes from the fact that the degeneracy $\bar{\omega}_{M}=\bar{\omega}_{M+1}$ corresponds to the maximum $\bar{\omega}$ with infinitesimally small curvature in the continuous case.

The $[N+1(\geq 4)]$ region model supports the exponential growth of disturbances with $m \geq 2$ and the algebraic growth of disturbances with $m=1$, consistent with some observations and numerical simulations. Since the $[N+1(\geq 4)]$-region model can be analytically treated more easily than the corresponding continuous model, it may be a useful tool to analytically investigate the behavior of asymmetric disturbances on hurricane-like vortices.

\section{Acknowledgments}

The authors would like to thank the two anonymous referees for their constructive criticism.

\section{References}

Chen, Y., and M. K. Yau, 2001: Spiral bands in a simulated hurricane. Part 1: Vortex Rossby wave verification. $J$. Atmos. Sci., 58, 2128-2145.

Flierl, T. G., 1988: On the stability of geostrophic vortices. $J$.
Fluid Mech., 197, 349-388.

Hoskins, B. J., M. E. McIntyre, and A. W. Robertson, 1985: On the use and significance of isentropic potential vorticity maps. Quart. J. Roy. Meteor. Soc., 111, 877946.

Mallen, K. J., and M. T. Montgomery, 2005: Reexamining the near-core radial structure of the tropical cyclone primary circulation: Implications for vortex resiliency. J. Atmos. Sci., 62, 408-425.

Montgomery, M. T., and R. J. Kallenbach, 1997: A theory of vortex Rossby waves and its application to spiral bands and intensity changes in hurricanes. Quart. J. Roy. Meteor. Soc., 123, 435-465.

Nolan, D. S., and M. T. Montgomery, 2000: The algebraic growth of wavenumber one disturbances in hurricanelike vortices. J. Atmos. Sci., 57, 3514-3538.

Reznik, G. M., and W. K. Dewar, 1994: An analytical theory of distributed axisymmetric barotropic vortices on the beta-plane. J. Fluid Mech., 269, 301-321.

Schecter, D. A., M. T. Montgomery, and P. D. Reasor, 2002: A theory for the vertical alignment of a quasigeostrophic vortex. J. Atmos. Sci., 59, 150-168.

Schubert, W. H., M. T. Montgomery, R. K. Taft, T. A. Guinn, S. R. Fulton, J. P. Kossin, and J. P. Edwards, 1999: Polygonal eyewalls, asymmetric eye contraction, and potential vorticity mixing in hurricanes. J. Atmos. Sci., 56, 1197-1223.

Smith, R. A., and M. N. Rosenbluth, 1990: Algebraic instability of hollow electron columns and cylindrical vortices. Phys. Rev. Lett., 64, 649-652.

Terwey, W. D., and M. T. Montgomery, 2002: Wavenumber2 and wavenumber- $m$ vortex Rossby wave instability in a generalized three-region model. J. Atmos. Sci., 59, 2421-2427.

Wang, Y., 2002: Vortex Rossby waves in a numerically simulated tropical cyclone. Part 1: Overall structure, potential vorticity, and kinetic energy budgets. J. Atmos. Sci., 59, 1213-1238. 